\title{
Estratégias de Vacinação: uma abordagem discreta
}

\author{
Priscila Roque de Almeida Mehran Sabeti \\ Universidade Federal de Viçosa - Departamento de Matemática \\ 36570-000, Viçosa, MG \\ E-mail: priscila.almeida@ufv.br,_mehran@ufv.br, \\ Lucy Tiemi Takahashi \\ Universidade Federal de Juiz de Fora - Departamento de Matemática \\ 36037-000, Juiz de Fora, MG \\ E-mail: 1tiemi@gmail.com.
}

\begin{abstract}
Resumo: Neste trabalho apresentaremos um estudo do modelo epidêmiológico SIR (Suscetíveis - Infectados - Recuperados), desenvolvido por McKendrick e Kermack em 1927, [3], que refere-se a doenças onde o indivíduo adquire imunidade após curar-se da infecção. Utilizando como base os modelos proposto por B. Shulgin et al., [4], no qual utilizam Equações Diferenciais para a modelagem de epidemias com estratégias de vacinação, utilizamos o método de aproximação de diferenças finitas para frente para discretizar estes sistemas contínuos afim de comparar os resultados e obter novos. Um aspecto importante da discretização é o ponto de vista distinto para estudar o modelo epidemiológico SIR, que aprova os modelos contínuos e é mais prático para aplicar os dados reais.
\end{abstract}

Palavras-chave: Epidemiologia, Modelo SIR, Modelos discretos, Vacinação constante, Vacinação em pulsos.

Uma das razões principais para o estudo de doenças infecciosas é melhorar o controle para tentar erradicá-las. Os modelos podem ser uma ferramenta poderosa nesta abordagem, o que nos permite otimizar o uso de recursos limitados ou simplesmente direcionar medidas de controle mais eficientes. Existem várias formas de medida de controle que funcionam de modo que reduz a quantidade média de transmissão entre indivíduos infecciosos e suscetíveis. Quais estratégia de controle, ou mistura de estratégias, serão utilizadas dependerá da doença, hospedeiros e dimensão da epidemia [2].

A maioria dos modelos epidêmicos baseia-se na divisão da população do hospedeiro em compartimentos, que são definidos levando em consideração as características ou propriedades físicas e epidemiológicas de cada doença específica. No presente estudo, consideramos as doenças infecciosas que podem ser modeladas pelo Modelo SIR, introduzido por Kermack e McKendrick, em 1927 [3]. Nele a população de hospedeiros é dividida em três compartimentos: Suscetíveis (S), indivíduos que não têm imunidade contra o agente infeccioso; Infectados (I), indivíduos que foram infectados e que podem transmitir a doença para indivíduos suscetíveis; e Recuperados (R), indivíduos que estão imunes à infecção.

Com base no trabalho proposto por B. Shulgin et al., [4], no qual utilizam Equações Diferenciais para a modelagem de epidemias com estratégias de vacinação, utilizamos o método de aproximação de diferenças finitas para frente para discretizar estes sistemas contínuos afim de comparar os resultados e obter novos. Um aspecto importante da discretização é o ponto de vista distinto para estudar o modelo epidemiológico SIR, que aprova os modelos contínuos e é mais prático para aplicar os dados reais.

Consideramos as seguintes hipóteses: i) todos os indivíduos nascem suscetíveis; ii) o tamanho da população é constante, para isso consideramos as taxas de natalidade e mortalidade constantes e iguais; iii) os indivíduos que se recuperam ganham imunididade; $i v$ ) a interação entre os componentes dá-se de forma homogônea; v) não há emigração e imigração do ambiente em estudo. Além disso, denotamos as taxas de natalidade e mortalidade, de contato e de recuperação por $m, \beta$ e $g$, respectivamente, sendo $0<m, g<1$ e $\beta>0$, e por $p$ a proporção de sucesso da vacinação, também com $0 \leq p \leq 1$. 
Denotamos a reprodutibilidade basal por $R_{0}$, onde $R_{0}=\frac{\beta}{m+g}$ - termo utilizado para designar o número de casos secundários da doenças gerados a partir de um caso primário. Para o estudo dos modelos com aplicação de estratégias de vacinação consideramos $R_{0}>1$.

Neste trabalho, para discretizar as equações contínuas, vamos utilizar a aproximação de diferenças finitas para frente, cuja expressão para a primeira derivada é dada por

$$
\frac{d S\left(t_{j}\right)}{d t}=\frac{S\left(t_{j+1}\right)-S\left(t_{j}\right)}{k}+\tau_{j}
$$

onde $k$ é o passo adotado e

$$
\tau_{j}=-\frac{k}{2} S^{\prime \prime}\left(\eta_{j}\right)+\cdots
$$

sendo $\eta_{j}$ um ponto entre $t_{j}$ e $t_{j+1}$. O termo $\tau_{j}$, como aparece em (2), indica o quão boa a aproximação está do problema inicial, sendo chamado erro de truncamento [1]. Utilizamos, para este trabalho, uma aproximação da equação diferencional ignorando o erro de truncamento, ou seja,

$$
\frac{d S\left(t_{j}\right)}{d t} \approx \frac{S\left(t_{j+1}\right)-S\left(t_{j}\right)}{k}
$$

e, como os parâmetros reais que podem ser obtidos são dados em dias, meses ou anos, utilizamos como passo uma unidade de tempo, $k=1$.

A estratégia de vacinação constante consiste em vacinar uma porcentagem $p$ de todos os recémnascidos, assim, considerando a população total normatizada para a unidade, ou seja, $S_{j}+I_{j}+R_{j}=1$, a proporção de indivíduos recém-nascidos suscetíveis diminui para $(1-p) m$, enquanto o compartimento dos indivíduos recuperados tem um aumento de $\mathrm{pm}$. Deste modo, discretizando o modelo contínuo sugerido em [4], obtemos o seguinte modelo de equações de diferenças finitas

$$
\left\{\begin{array}{c}
S_{j+1}=S_{j}+(1-p) m-\beta I_{j} S_{j}-m S_{j} \\
I_{j+1}=I_{j}+\beta I_{j} S_{j}-m I_{j}-g I_{j} \\
R_{j+1}=R_{j}+p m+g I_{j}-m R_{j},
\end{array}\right.
$$

onde $0 \leq S_{j}, I_{j}, R_{j} \leq 1$ são proporções de indivíduos suscetíveis, infectados e recuperados no tempo $j$, respectivamente.

A análise da estabilidade dos pontos de equilíbrio para este modelo revela que, se $0<\beta<1$, assim como acontece para o modelo contínuo, há uma proporção mínima de vacinação, denotada $p_{c}$ e dada por $p_{c}=1-\frac{1}{R_{0}}$, que governa a dinâmica do sistema da seguinte forma: se $p>p_{c}$, ou seja, a taxa de vacinação for maior que a taxa mínima de vacinação, então o ponto de equilíbrio livre de infecção, $P_{0}=(1-p, 0)$, é estável. Para a análise do ponto de equilíbrio epidêmico, realizada numericamente, verificamos que se $p<p_{c}$, ou seja, se a taxa de vacinação for relativamente baixa, então o ponto de equilíbrio epidêmico $P_{1}=\left(\frac{m+g}{\beta}, m\left[\frac{1-p}{m+g}-\frac{1}{\beta}\right]\right)$, é estável.

Em vez de vacinar constantemente uma proporção extremamente grande de todos os recém nascidos suscetíveis, o programa de vacinação em pulsos propõe vacinar uma fração $p$ de toda a população de suscetíveis em um único pulso, aplicado a cada $T$ anos [4]. Assim, o modelo contínuo sugerido pelos autores é dado por

$$
\left\{\begin{array}{l}
\frac{d S}{d t}=m-\beta I S-m S-p \sum_{n=0}^{\infty} S\left(n T^{-}\right) \delta(t-n T) \\
\frac{d I}{d t}=\beta I S-m I-g I
\end{array}\right.
$$

onde os parâmetros são os mesmos já definidos acima, $S\left(n T^{-}\right)=\lim _{\epsilon \rightarrow 0} S(n T-\epsilon)$ é o número de indivíduos suscetíveis imediatamente antes o $n$-ésimo pulso de vacinação e $\delta(t-n T)$ é a função Delta de Dirac, dada por

$$
\delta(t-n T)=\left\{\begin{array}{r}
0, \text { se } t \neq n T \\
\infty, \text { se } t=n T
\end{array}\right.
$$


$\operatorname{com} \int_{-\infty}^{\infty} \delta(t-n T)=1$

De acordo com Shulgin et al., [4], a estratégia de vacinação em pulsos deve ser aplicada sempre que a população suscetível, $S(t)$, cresce a um nível muito próximo do limiar epidêmico, $S_{c}=\frac{1}{R_{0}}$. No "método do limiar", os pulsos periódicos podem manter $S(t)$ abaixo de $S_{c}$ desde que o período entre os pulsos, $T$, seja mantido abaixo de um valor crítico fixado, denotado por $T_{\mathrm{Max}}$ cuja melhor aproximação é $T_{\text {Max }}=\frac{p g}{m \beta} \frac{1}{\left(1-\frac{p}{2}-\frac{g}{\beta}\right)}$. Obviamente, quanto maior o período entre os pulsos de vacinação, menos vezes a população é vacinada. No entanto, se este período exceder o valor crítico, $T_{M a x}$, o programa de vacinação pode ser falho

Para discretizar este modelo, vamos separá-lo em dois casos: $i$ ) Quando $t \neq n T$ não haverá vacinação, então a discretização do modelo é dada por:

$$
\left\{\begin{array}{l}
S_{j+1}=S_{j}+m-\beta I_{j} S_{j}-m S_{j} \\
I_{j+1}=I_{j}+\beta I_{j} S_{j}-m I_{j}-g I_{j}
\end{array}\right.
$$

ii) Quando $t=n T$, uma proporção $p$ de todos os indivíduos suscetíveis é vacinada, de onde obtemos o seguinte modelo discreto

$$
\left\{\begin{array}{l}
S_{n T}=m+(1-p) S_{n T-1}-\beta I_{n T-1} S_{n T-1}-m S_{n T-1} \\
I_{n T}=I_{n T-1}+\beta I_{n T-1} S_{n T-1}-(m+g) I_{n T-1} .
\end{array}\right.
$$

O estudo sobre a estabilidade deste modelo é feito por meio das simulações numéricas apresentadas a seguir.

Consideramos como parâmetros $m=0,004, g=0,1429, \beta=0,90$ e como condição inicial $\left(S_{0}, I_{0}\right)=(0,90,0,10)$. Inicialmente, supondo que a cobertura de cada pulso de vacinação seja de $10 \%$ da população e aplicando a vacinação com intervalo de 4 dias entre os pulsos, observamos que a população de indivíduos infectados torna-se extinta, Figura 1.

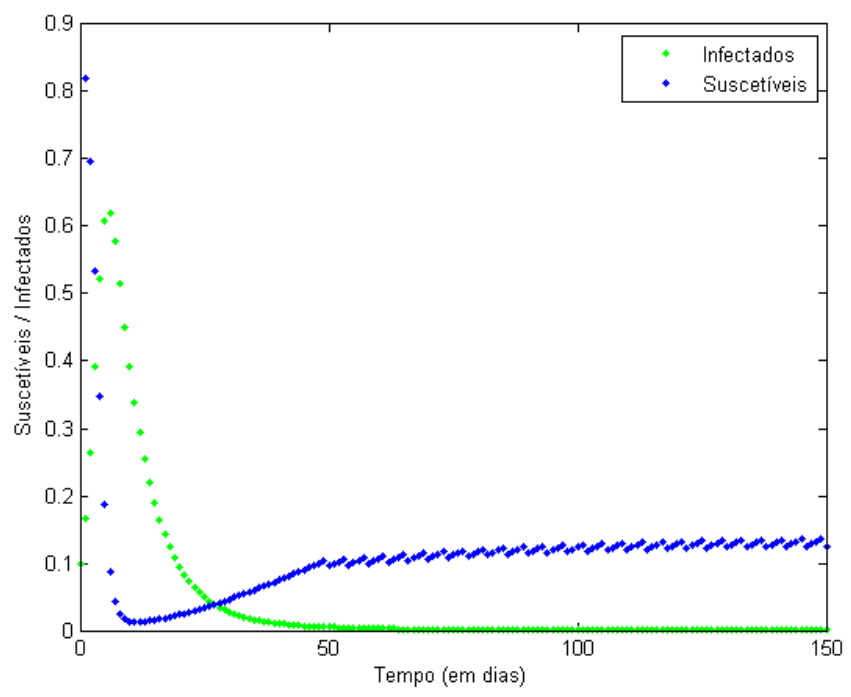

Figura 1: Dinâmica entre as populações de indivíduos suscetíveis e infectados considerando $m=0,004$, $g=0,1429, \beta=0,90, p=0,10,\left(S_{0}, I_{0}\right)=(0,90,0,10)$ e $T=4$. Simulação realizada com intervalo de 150 dias.

Aumentando o intervalo entre os pulsos de vacinação para 20 dias verificamos, Figura 2, que não há extinção e após certo tempo a população de infectados torna a crescer, entrando em equilíbrio epidêmico. 


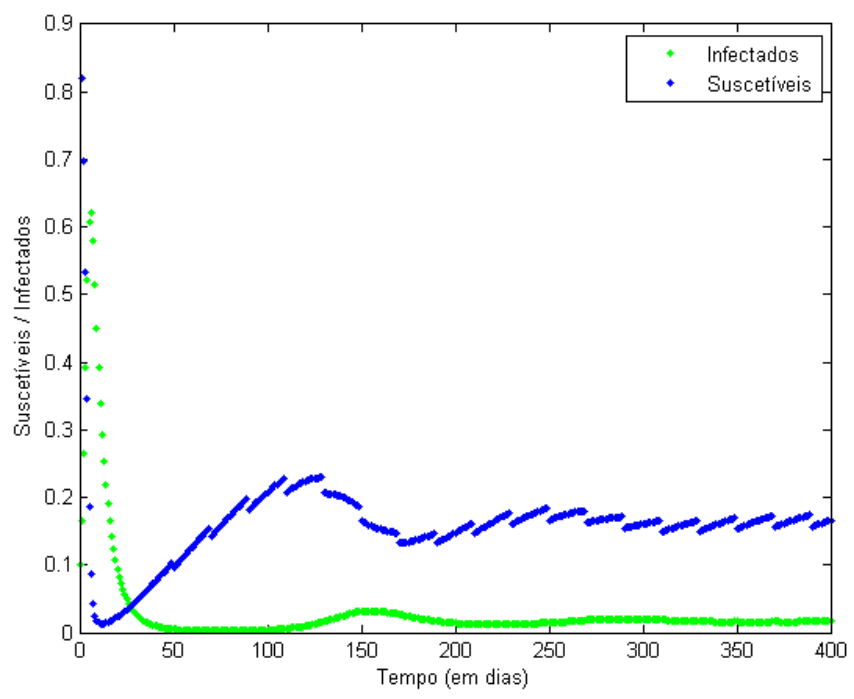

Figura 2: Dinâmica entre as populações de indivíduos suscetíveis e infectados considerando como parâmetros $m=0,004, g=0,1429, \beta=0,90, p=0,10,\left(S_{0}, I_{0}\right)=(0,90,0,10)$ e $T=20$. Simulação realizada com intervalo de 400 dias.

Posteriormente, aumentando a cobertura de vacinação no modelo discreto para $40 \%$ dos indivíduos da suscetíveis da população e aplicando a vacinação a cada 20 dias observamos o desaparecimento da doença, Figura 3.

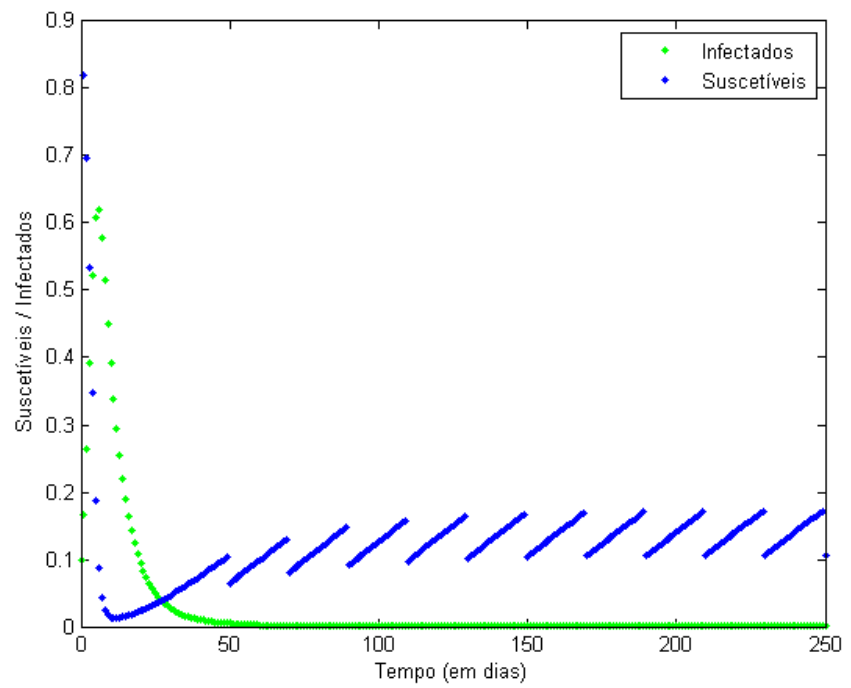

Figura 3: Dinâmica entre as populações de indivíduos suscetíveis e infectados considerando como parâmetros $m=0,004, g=0,1429, \beta=0,90, p=0,40,\left(S_{0}, I_{0}\right)=(0,90,0,10)$ e $T=20$. Simulação realizada com intervalo de 250 dias. 
Mantendo a proporção de vacinação, $p=0,40$, e alterando o intervalos entre os pulsos para 35 dias, Figura 4, observamos que a população de infectados começa a oscilar após determinado tempo e não é extinta.

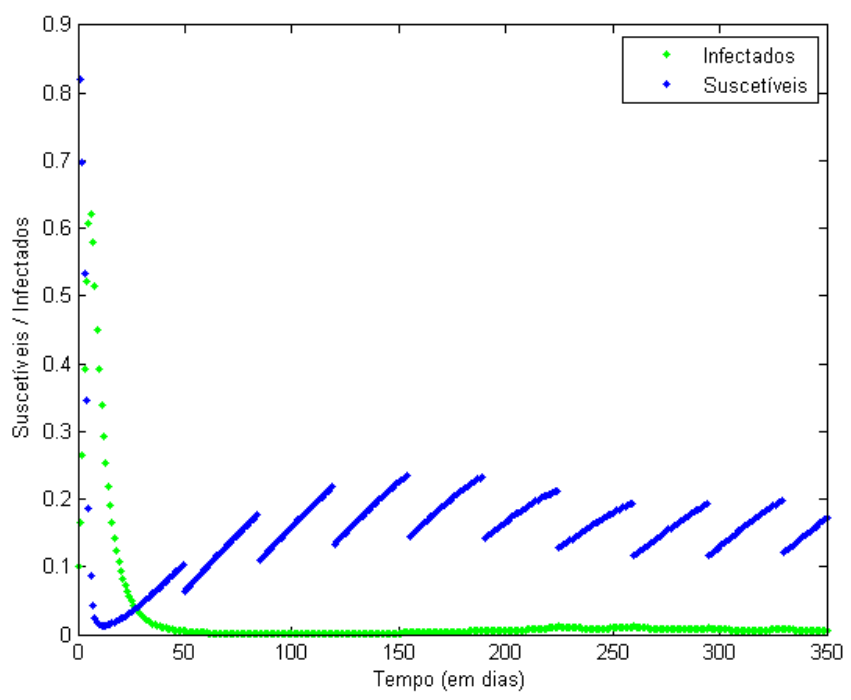

Figura 4: Dinâmica entre as populações de indivíduos suscetíveis e infectados considerando como parâmetros $m=0,004, g=0,1429, \beta=0,90, p=0,40,\left(S_{0}, I_{0}\right)=(0,90,0,10)$ e $T=35$ Simulação realizada com intervalo de 350 dias.

Como demonstrado para o modelo contínuo, para o modelo discreto SIR com vacinação em pulsos também existe um intervalo de tempo máximo que garante a estabilidade das soluções. Caso o intervalo entre os pulsos de vacinação seja maior que este $T_{\mathrm{Max}}$ calculado não há extinção da doença. Além disso, a aproximação para a expressão do $T_{\text {Max }}$ também é válida para o modelo discreto. Assim, verificamos que mantendo o período entre os pulsos de vacinação abaixo do período máximo $T_{\mathrm{Max}}=\frac{p g}{m \beta} \frac{1}{\left(1-\frac{p}{2}-\frac{g}{\beta}\right)}$, sugerido para o modelo contínuo por Shulgin et al., [4], obtemos o equilíbrio livre de infecção para o modelo discreto com vacinação em pulsos.

A programação de modelos discretos é mais simples do que para modelos contínuos, deste modo a discretização dos problemas contínuos facilita sua programação e simulação gráfica, o que resulta em um menor gasto computacional. Realizando uma discretização dos modelos epidemiológicos contínuos, verificamos que os resultados encontrados para os modelos discretos são muito próximos àqueles encontrados para os modelos contínuos. Assim, apesar da aproximação feita, o erro de truncamento não afeta o resultado esperado. Para um trabalho futuro, reduziremos o passo de discretização, que foi de uma unidade de tempo, para tentar eliminar a hipótese extra necessária para garantir a estabilidade no modelo com vacinação constante, $0<\beta<1$, e coletaremos dados reais, de campanhas de vacinação, afim de aperfeiçoarmos e validarmos estes modelos. Considerando esta validação, poderemos fazer previsões sobre a evolução de determinada epidemia com mais facilidade e montar estratégias de vacinação capazes de gerar economia de recursos.

\section{Referências}

[1] HOLMES, M. H., "Introduction to Numerical Methods in Differential Equations". New York: Springer-Verlang, 2000.

[2] M. J. Keeling, P. Rohani, "Modeling Infectious Diseases in Humans and Animals", Princeton: Princeton University Press, 2011. 
[3] W. Kermack, A. McKendrick, A contribution to the mathematical theory of epidemics, em "Proceedings of the Royal Society of London", Series A, Mathematical and Physical Sciences, A115: 700-721, 1927.

[4] B. Shulgin, L. Stone, Z. Agur, Pulse Vaccination Strategy in the SIR Epidemic Model, em "Bulletin Mathematical Biology", Vol. 60, pp. 1123-1148, 1998. 